\title{
Fraunhofer regime of operation for superconducting quantum interference filters
}

Shadrin, A.V.; Constantinian, K.Y.; Ovsyannikov, G.A.; Shitov, S.V.; Soloviev, I.I.; Kornev, V.K.; Mygind, Jesper

Published in:

Applied Physics Letters

Link to article, DOI:

$10.1063 / 1.3058759$

Publication date:

2008

Document Version

Publisher's PDF, also known as Version of record

Link back to DTU Orbit

Citation (APA):

Shadrin, A. V., Constantinian, K. Y., Ovsyannikov, G. A., Shitov, S. V., Soloviev, I. I., Kornev, V. K., \& Mygind, J. (2008). Fraunhofer regime of operation for superconducting quantum interference filters. Applied Physics Letters, 93(26), 262503. https://doi.org/10.1063/1.3058759

\section{General rights}

Copyright and moral rights for the publications made accessible in the public portal are retained by the authors and/or other copyright owners and it is a condition of accessing publications that users recognise and abide by the legal requirements associated with these rights.

- Users may download and print one copy of any publication from the public portal for the purpose of private study or research.

- You may not further distribute the material or use it for any profit-making activity or commercial gain

- You may freely distribute the URL identifying the publication in the public portal 


\title{
Fraunhofer regime of operation for superconducting quantum interference filters
}

\author{
A. V. Shadrin ${ }^{1, \text { a) }}$ K. Y. Constantinian, ${ }^{1}$ G. A. Ovsyannikov, ${ }^{1}$ S. V. Shitov, ${ }^{1}$ I. I. Soloviev, ${ }^{2}$ \\ V. K. Kornev, and J. Mygind ${ }^{3}$ \\ ${ }^{1}$ Kotel'nikov Institute of Radio Engineering and Electronics, Russian Academy of Sciences, \\ Moscow 125009, Russia \\ ${ }^{2}$ Department of Physics, Moscow State University, Moscow 119992, Russia \\ ${ }^{3}$ Institute of Physics, Technical University of Denmark, DK-2800 Kgs. Lyngby, Denmark
}

(Received 21 July 2008; accepted 7 December 2008; published online 30 December 2008)

\begin{abstract}
Series arrays of superconducting quantum interference devices (SQUIDs) with incommensurate loop areas, so-called superconducting quantum interference filters (SQIFs), are investigated in the kilohertz and the gigahertz frequency range. In SQIFs made of high- $T_{c}$ bicrystal junctions the flux-to-voltage response $\partial V / \partial \Phi$ is dominated by the variation in the critical current in the individual junctions (Fraunhofer-type) rather than by the SQUIDs interference. For a SQIF with 20 SQUID loops we find $\partial V / \partial \Phi=40 \mathrm{mV} / \Phi_{0}$ and a dynamic range of more than $60 \mathrm{~dB}$ in the kilohertz range. In the 1-2 GHz range the estimated power gain is $20 \mathrm{~dB}$ and the magnetic flux noise level is as low as $10^{-4} \Phi_{0}$. (C) 2008 American Institute of Physics. [DOI: 10.1063/1.3058759]
\end{abstract}

Microwave amplifiers based on superconducting quantum interference effects in two parallel connected Josephson junctions (JJs) (SQUID) (SQUID denotes superconducting quantum interference device) are characterized by a noise temperature close to the quantum limit (see, e.g., Refs. 1 and 2). SQUID amplifiers, as well as other Josephson devices without feedback, posses low saturation power, which is proportional to the characteristic voltage $V_{0}=I_{c} R_{n}$, where $I_{c}$ is the critical current and $R_{n}$ is the normal state resistance of the JJ. For resistively shunted tunnel JJs made of low- $T_{c}$ superconductors (LTS), $V_{0}$ (here $R_{n}$ is the shunt resistance needed to avoid hysteresis) only reaches $200-300 \mu \mathrm{V}$ at $T$ $=4.2 \mathrm{~K}$ (Ref. 2), while the bicrystal JJs made of high- $T_{c}$ superconductors (HTS) can give $V_{0}=1 \mathrm{mV}$ at $T=77 \mathrm{~K}^{3}{ }^{3}$

The output signal and saturation power can be increased by using an array of JJs or SQUIDs. An increase in the output signal proportional to the number $N$ of SQUIDs was demonstrated in an amplifier based on a series-connected array of LTS SQUIDs. ${ }^{4}$ Generally, however, the spread in $I_{c}$ and $R_{n}$ parameters of the HTS JJs is a pertinent problem that restricts the use of series-connected JJs or SQUID arrays. Superconducting quantum interference filters (SQIFs) arrays of SQUIDs (series or parallel connected) with incommensurate SQUID-loop areas-accept much wider margins in parameter spread. ${ }^{5-7}$ For a SQIF with small normalized array loop inductances $l_{i}<1$ (where $l_{i}=2 \pi I_{c} L_{i} / \Phi_{0}, \Phi_{0}$ $=h / 2 e$ is the magnetic flux quantum, $I_{c}$ is the critical current, and $L_{i}$ is the SQUID-loop inductance) and a suitably chosen distribution of loop sizes, the magnetic field to voltage response $V(H)$ is a nonperiodic function with a single narrow global minimum at $H=0$. The contributions from the different SQUID loops are washed out, and the width of the $V(H)$ minimum is determined by the number of loops and the size of the largest SQUID loop. ${ }^{7}$ For short we will call this operational regime of the SQIF with a single $V(H)$ minimum at $H=0$ the $S$-mode. Note that in the $S$-mode also the influence of the Fraunhofer-like $I_{c}(H)$ dependence in the individual JJs

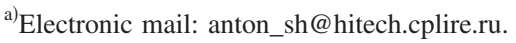

is visible but may be neglected. Recently, HTS SQIFs have been studied for microwave amplification. ${ }^{8,9}$

Sensitive magnetometers based on one-dimensional series arrays of HTS bicrystal JJs have been reported. ${ }^{10}$ Here both the Fraunhofer dependence of the critical current in the $\mathrm{JJs}_{\mathrm{s}} I_{c}(H)=I_{c}(0)\left|\sin \left(\pi \Phi_{J} / \Phi_{0}\right) /\left(\pi \Phi_{J} / \Phi_{0}\right)\right|\left(\right.$ where $\Phi_{J}$ $=\mu_{0} H a_{J}$ is the magnetic flux in a $\mathrm{JJ}$ with effective area $a_{J}$ ), and the flux focusing due to the geometry of the bicrystal JJ have to be taken into account. We call this operational regime the $F$-mode. A field-voltage transfer function $d V / d \mu_{0} H=7500 \mathrm{~V} / \mathrm{T}$ ( $\mu_{0}$-vacuum permeability) was obtained for an array with $N=105$ series-connected JJs. ${ }^{10}$ This compares well with the sensitivity of a LTS SQUID array with small spread of parameters. ${ }^{4}$

In this paper we investigate series-connected SQIF consisting of 20 HTS bicrystal SQUIDs with SQUID-loop inductances in the range of $15-300 \mathrm{pH}$. The width ( $w$ $=10 \mu \mathrm{m})$ of the film forming both the JJs and the SQUID loops is much larger than the London penetration depth $\lambda_{L}$ in $\mathrm{YBa}_{2} \mathrm{Cu}_{3} \mathrm{O}_{x}$ (YBCO). The external magnetic flux produced by the input signal coil induces circulating currents in the superconducting SQUID loops. Since $w \gg \lambda_{L}$ the current circulating along the inside edge of the SQUID loop is compensated by the current in the outer edge of the SQUID loop. The presence of the circulating current in the JJs provides better inductive coupling between the JJs and the input circuit and in turn increases the microwave amplification.

Bicrystal $\mathrm{NdGaO}_{3}$ (NGO) substrates were used in the sample fabrication. NGO is characterized by a tolerable permittivity $(\varepsilon=25)$ and fairly low microwave $\operatorname{losses}(\operatorname{tg} \delta$ $\left.<10^{-3}\right)$. Devices were formed by ion-plasma and chemical etching of YBCO film deposited by dc sputtering at high oxygen pressure. The fabrication details of bicrystal JJs have been described elsewhere. ${ }^{3}$ Single loop SQUID and seriesconnected SQUID arrays ${ }^{8}$ were fabricated for comparison. Figure 1 shows the topology of a SQIF designed for use as microwave amplifier. The input line (Au film) was deposited over a $\mathrm{SiO}_{2}$ insulator layer; the bottom layer is the $\mathrm{YBCO}$ film that forms the SQIF located inside the input line. The 


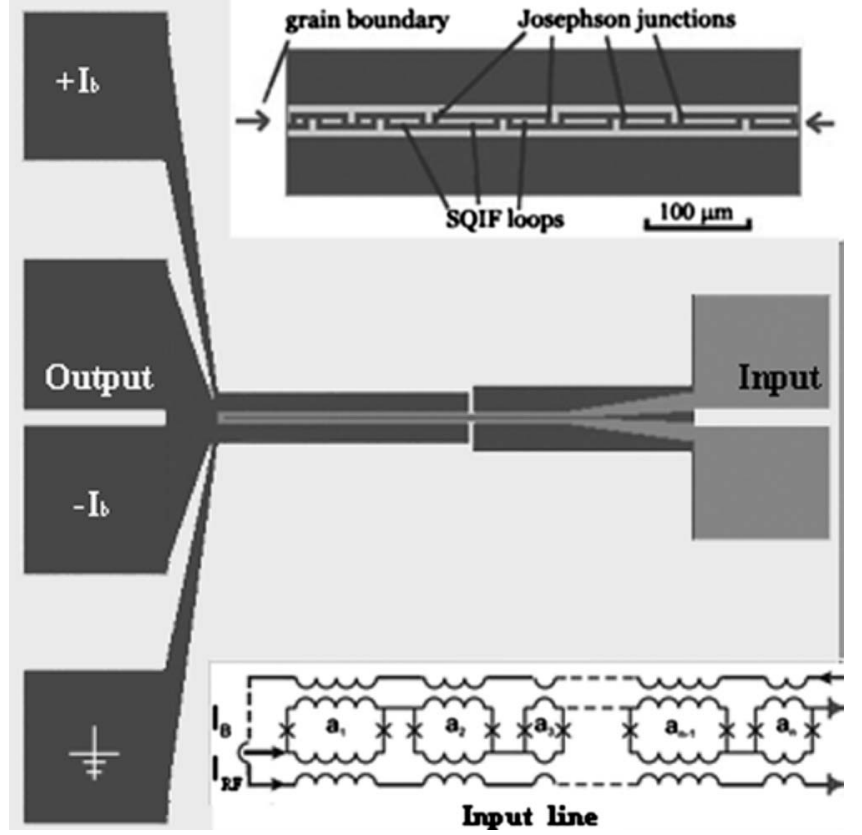

FIG. 1. Layout of a SQIF designed for microwave amplifier. It consists of 20 series-connected SQUID loops with areas in the range of 35-700 $\mu \mathrm{m}^{2}$. The width $w$ of the JJs is $10 \mu \mathrm{m}$. The input line circuit for the $I_{\mathrm{rf}}$ current consists of a top $\mathrm{Au}$ thin film (gray color) deposited over the $\mathrm{SiO}_{2}$ buffer layer. The YBCO thin film is the bottom layer (dark color). The output circuit is a coplanar-line YBCO film with the SQIF junctions located along the bicrystal boundary. The top inset shows a zoomed view of the bottom layer with a part of the SQIF. The bottom inset shows a circuit with a SQIF and pick-up loop. $I_{b}$ is dc bias current.

output voltage signal is taken directly from the SQIF.

We measured dc $I-V$ and $V(H)$ curves at low frequency as well as the output noise power $P_{n}=k_{b} T_{n} \Delta f$ in the frequency range $f=1-2 \mathrm{GHz}$. For the noise power measurements we used a cryogenic preamplifier with noise temperature $T_{\mathrm{A} 1}=(8 \pm 2) \mathrm{K}$ and gain $G=21 \mathrm{~dB}$, followed by a room temperature amplifier with $T_{\mathrm{A} 2}=130 \mathrm{~K}$ and $G=40 \mathrm{~dB}$. The output voltage signal was simultaneously recorded by a spectrum analyzer and a quadratic detector integrated into the room amplifier.

From the $I_{c}(H)$ pattern measured on the reference SQUID with loop area $S=35 \mu \mathrm{m}^{2}$ we estimate a loop inductance $L=(15 \pm 5) \mathrm{pH}^{8,11}$ The estimated spread in critical currents of JJs in the SQIF was $\delta I / I_{n} \approx 30 \%$. Using the mean value of $I_{c}=100 \mu \mathrm{A}$ for JJs with width $w=10 \mu \mathrm{m}$, we calculate the normalized inductances of the loops in the SQIF to be in the range $l_{i}=4-90$.

Numerical simulations (using the PSCAN program) ${ }^{12}$ of the $V(H)$ curve for SQIFs with different number of loops $N$ are shown in Fig. 2. Experimental values of loop inductances as well as the Fraunhofer dependence of the JJ critical currents are taken into account. This leads to an aperiodic $V(H)$ response even for a single SQUID. The modulation of the side lobes is suppressed with increasing $N$. Dips due to both Fraunhofer modulations in junctions and SQUID interference can be seen for $N>10$. Finally for $N=20$ one observes a wide dip due to the Fraunhofer dependence of the critical currents of the JJs ( $F$-mode) and a small, narrow zero dip due to the effect of the SQUID loops ( $S$-mode). Note that taking into account a 30\% spread of the critical currents $I_{c i}$ of the JJs in the SQIF, the numerical simulation gives a decrease in both $F$ - and $S$-mode amplitudes.

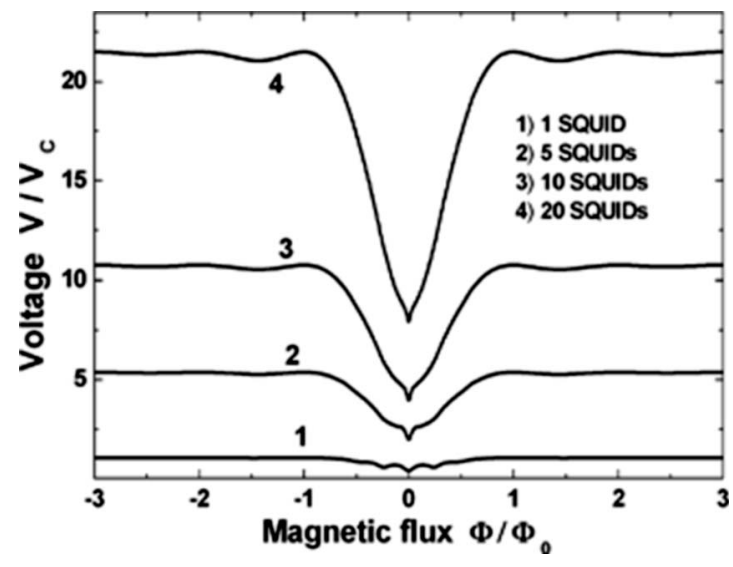

FIG. 2. Numerical simulation of the $V(H)$ response for SQIFs with different numbers of loops. The Fraunhofer dependence of the JJ critical currents is taken into account. For the SQIF the normalized inductances are in the range $l_{i}=4-90$. For the single SQUID $l_{i}=4$. Dips due to both Fraunhofer modulation in the junctions and the SQUID interference can be seen for $N>10$.

Figure 3 shows a family of $V(H)$ responses of a SQIF with 20 loops plotted at different dc bias currents $I_{b}$. A single $F$-mode dip with small side voltage modulation is seen for all $I_{b}$. The inset shows a zoom-in of the central part. Besides the single $S$-mode dip predicted by numerical simulations (Fig. 2), additional dips produced by the SQUIDs response interference are observed. The width of the $V(H)$ dip is considerably larger than the dip in a SQIF operated in the $S$-mode. ${ }^{5-7,9}$ The width of the $F$-mode dip is well fitted using an effective JJ area $S_{\text {eff }}=30 \mu \mathrm{m}^{2}$. This is larger than the evaluated area of $\mathrm{JJ} w \lambda_{L}=1.5 \mu \mathrm{m}^{2}$, where $w$ is the JJ width and $\lambda_{L}=0.15 \mu \mathrm{m}$ is the London penetration depth in YBCO. This observation is in agreement with a strong flux focusing effect in a bicrystal JJ (Ref. 13) when the magnetic field is applied perpendicular to the film. The expected value of the $V(H)$ response, $V_{S}=\Sigma V_{0 i}$, should increase with the number $N$ of SQIF loops, each contributing $V_{0 i}=I_{c i} R_{n i}$. However, the experimental $V_{S}=12 \mathrm{mV}$ turned out smaller than the estimated $\Sigma V_{0 i}=20 \mathrm{mV}$ for a SQIF with $N=20$. For the SQIF

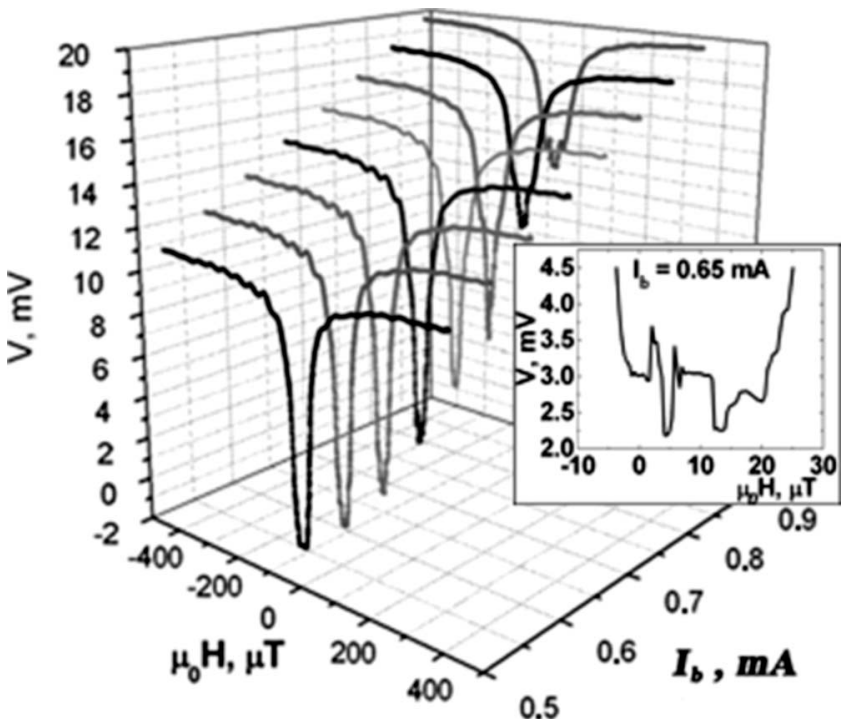

FIG. 3. Magnetic field-to-voltage response $V(H)$ of a SQIF array with 20 loops for different dc bias currents $I_{b}$. The measurements were made at $T=4.2 \mathrm{~K}$. For the structure with critical current $I_{c}=560 \mu \mathrm{A}$, the maximum amplitude of $d V / d H$ is observed at $I_{b}=1.1 I_{c}$. The fine structure of the central part of the $F$-mode dip is shown in the inset. 


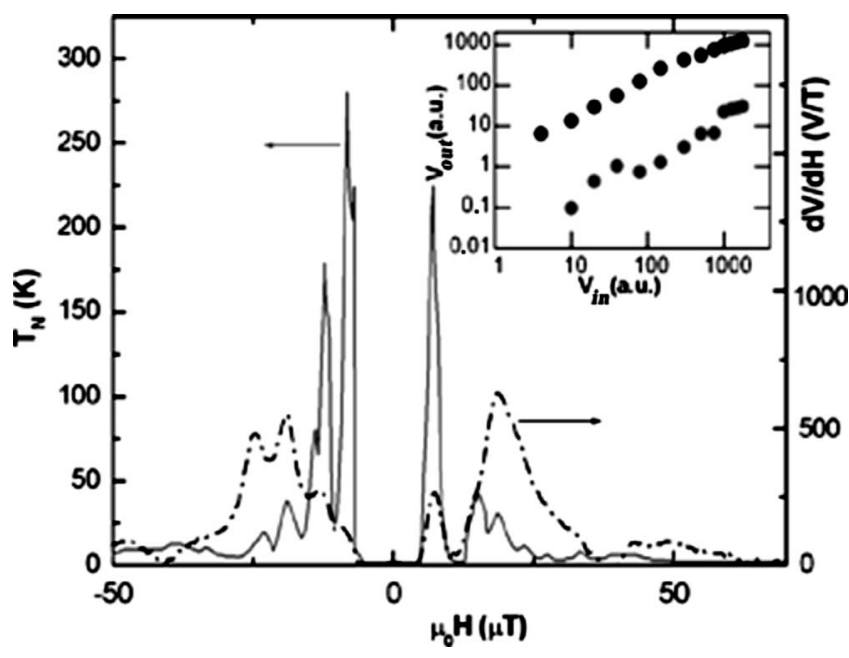

FIG. 4. Magnetic field dependence of the noise temperature $T_{n}(H)$ for a 20 loop SQIF in the frequency band $f=1-2 \mathrm{GHz}$ (solid line). The dash-dotted line shows the $d V / d H(H)$ dependence. The bias current is $I_{b}=1.25 I_{c}$, $T=4.2 \mathrm{~K}$. The inset shows the dependence of the first (black points) and second harmonics (gray points) of the output signal $V_{\text {out }}$ vs input signal $V_{\text {in }}$ at frequency $f_{\text {in }}=900 \mathrm{~Hz}$.

operating in the $F$-mode we obtain $d V / d \Phi=40 \mathrm{mV} / \Phi_{0}$ (and $\left.d V / d \mu_{0} H=270 \mathrm{~V} / \mathrm{T}\right)$, while for the reference SQUID $d V / d \Phi=1 \mathrm{mV} / \Phi_{0}{ }^{8}$. Thus for a SQIF made of HTS bicrystal JJs, the $F$-mode plays a predominant role in the $V(H)$ response.

The output noise temperature $T_{n}(H)$ measured in the frequency range $f=1-2 \mathrm{GHz}$ for a 20 loop SQIF is presented in Fig. 4. The output noise power is up to $15 \mathrm{~dB}$ above the background noise level. The measured output noise signal from the SQIF can be interpreted as an incoherent superposition of voltage spectral density of thermal fluctuations $S_{V}^{T}$ $=\sum\left(8 k T R_{d}^{2} / R_{n}\right)\left[1+1 / 2\left(I / I_{c}\right)^{2}\right]$ and voltage spectral density corresponding to contribution of magnetic flux conversion $S_{V}^{\Phi}=\Sigma\left(2 k T L_{n}^{2} / R_{n}\right)\left(d V_{n} / d \Phi_{n}\right)^{2} \cdot{ }^{14,15}$ For a bias current $I_{b}$ $=1.25 I_{c}$ and $R_{d}=30 \Omega$ at the $T_{n}(H)$ peak we get $S_{V}^{\Phi} / S_{V}^{T}$ proportional to $\max \left(l_{i}\right) / N>1$ [neglecting $R_{d i}(H)$ and $I_{c i}$ variation in the SQIF]. It indicates that the observed output noise signal is dominated by the magnetic flux conversion $S_{V}^{\Phi}$ rather than the thermal fluctuations $S_{V}^{T}$. With $d V / d \Phi$ $=40 \mathrm{mV} / \Phi_{0}$, the SQIF is characterized by a flux sensitivity $\delta \Phi=10^{-4} \Phi_{0}$. Both the $d V / d H(H)$ and the $T_{n}(H)$ functions have complex dependences with several maxima. However, some of the $T_{n}(H)$ peaks are correlated with the $d V / d H$ function. The side lobe modulation of the $d V / d H$ [weakly seen in the $V(H)$ response] and the corresponding behavior of the $T_{n}(H)$ function may be caused by residual contributions from the SQUID-loop interference that could be increased by the asymmetry in the critical currents in large inductive SQUIDs $(l \gg 1) .{ }^{16}$ Finally the complex behavior of the $T_{n}(H)$ function could be the result of the $R_{d}(H)$ dependence due to the scattering of the $I_{c i}$ parameters.

In order to estimate the power gain $G$ $=M^{2}(d V / d \Phi)^{2} / R_{d} R_{s},{ }^{17,18}$ we take $R_{s}=50 \Omega$ as the microwave source resistance, the measured dynamic resistance $R_{d}=30 \Omega$, and an estimated mutual coupling inductance $M=2.4 \times 10^{-11} \mathrm{H}$, assuming a geometrical coupling coefficient $\alpha=0.2$ (for the SQIF layout shown in Fig. 1). Using the experimental value $d V / d \Phi=2 \times 10^{13} \mathrm{~s}^{-1}$ obtained from measurements at low frequencies, we get a power gain $G=20 \mathrm{~dB}$.

In order to estimate the saturation power of the SQIF array, we measured the output signal at low frequencies $f=49 \mathrm{~Hz}-90 \mathrm{kHz}$. The expected saturation power of noncoherently operating SQIF loops increases as the square root of $N, P_{S} \propto \sqrt{N} .^{5-7}$ An analysis of the experimental data for the first and the second harmonic of the output signal response with an applied $900 \mathrm{~Hz}$ signal to the 20 loop SQIF biased at $I / I_{c}=1,1$ shows quasilinear dependence over $60 \mathrm{~dB}$ (see the inset in Fig. 4). We observed a signal distortion with the second harmonic amplitude of about $1 \%$ relative to of the first harmonic amplitude. Note that a similar harmonic distortion was reported for a LTS SQUID amplifier. ${ }^{18}$

Summarizing, we have fabricated and studied HTS SQIFs operating in the $F$-mode where the magnetic field-tovoltage response is mostly determined by a Fraunhofer dependence of the critical current of the individual JJs. The flux-to-voltage conversion factor in the $F$-mode is apparently lower than the factor expected in the originally suggested $S$-mode due to the smaller effective areas of the JJs than of the SQUID loops. Nevertheless our SQIF showed a power gain $G>1$ in the $F$-mode and a significant increase in saturation power and dynamic range.

The authors thank I. V. Borisenko, Yu. V. Kislinski, A. V. Kolabukhov, P. V. Komissinskiy, I. M. Kotelynski, A. V. Sobolev, and D. Winkler for fruitful discussions. This work was partially supported by Scientific School (Grant No. NSh5008.2008.2), FP6 European Union program (Grant No. NMP3-CT-2006-033191), RFBR (Grant No. 08-02-00487), ESF program AQDJJ and ISTC Project 3743.

${ }^{1}$ M. Mück, Ch. Welzel, and J. Clarke, Appl. Phys. Lett. 82, 3266 (2003). ${ }^{2}$ G. V. Prokopenko, S. V. Shitov, I. L. Lapitskaya, V. P. Koshelets, and J. Mygind, IEEE Trans. Appl. Supercond. 13, 1042 (2003).

${ }^{3}$ I. V. Borisenko, I. M. Kotelyanski, A. V. Shadrin, P. V. Komissinski, and G. A. Ovsyannikov, IEEE Trans. Appl. Supercond. 15, 165 (2005).

${ }^{4}$ M. E. Huber, P. A. Neil, R. G. Benson, D. A. Burns, A. M. Corey, C. S. Flynn, Y. Kitaygorodskaya, O. Missihzadeh, J. M. Martinis, and G. C. Hilton, IEEE Trans. Appl. Supercond. 11, 4048 (2001).

${ }^{5}$ J. Oppenländer, P. Caputo, Ch. Häussler, T. Träuble, J. Tomes, and A. Friesch, Appl. Phys. Lett. 85, 989 (2001).

${ }^{6}$ V. Schultze, R. IJsselsteijn, and H.-G. Meyer, Supercond. Sci. Technol. 19, S411 (2006).

${ }^{7}$ Ch. Häussler, J. Oppenländer, and N. Schopohl, J. Appl. Phys. 89, 1875 (2001).

${ }^{8}$ A. V. Shadrin, K. Y. Constantinian, and G. A. Ovsyannikov, Tech. Phys. Lett. 33, 192 (2007).

${ }^{9}$ O. V. Snigirev, M. L. Chukharkin, A. S. Kalabukhov, M. A. Tarasov, A. A. Deleniv, O. A. Mukhanov, and D. Winkler, IEEE Trans. Appl. Supercond. 17, 718 (2007).

${ }^{10}$ S. Krey, O. Brugmann, and M. Schilling, Appl. Phys. Lett. 74, 293 (1999)

${ }^{11} \mathrm{H}$. Hasegawa, Y. Tarutani, T. Fukazawa, and K. Takagi, IEEE Trans. Appl. Supercond. 8, 26 (1998).

${ }^{12}$ V. K. Kornev and A. V. Arzumanov, Inst. Phys. Conf. Ser. 158, 627 (1997)

${ }^{13}$ P. A. Rosenthal and M. R. Beasley, Appl. Phys. Lett. 59, 3482 (1991).

${ }^{14}$ C. D. Tesche and J. Clarke, J. Low Temp. Phys. 29, 301 (1977).

${ }^{15}$ K. Enpuku, G. Tokida, T. Maruo, and T. Minotani, J. Appl. Phys. 78, 3498 (1995).

${ }^{16}$ G. Testa, E. Sarnelli, S. Pagano, C. R. Calidonna, and M. Mango Furnari, J. Appl. Phys. 89, 5145 (2001)

${ }^{17}$ C. Hilbert and J. Clarke, J. Low Temp. Phys. 61, 263 (1985).

${ }^{18}$ M. Mück and J. Clarke, Appl. Phys. Lett. 78, 3666 (2001). 